\title{
Sexually transmitted diseases: an epidemic in adolescent girls?
}

\author{
GRETA E FORSTER, GILLIAN E ROBINSON, AND PATRICIA E MUNDAY \\ From the Praed Street Clinic, St Mary's Hospital, London
}

SUMMARY The prevalence of sexually transmitted disease (STD) in adolescent girls aged 15-19 attending a department of genitourinary medicine was compared for the years 1972 and 1982. Data were obtained from the confidential register using the coding of the clinic returns to the Department of Health (form SBH 60). There were 1373 patients in 1972 and 1799 in 1982, 6.4\% and $7 \%$ respectively of the total female clinic population. The prevalence of syphilis, gonorrhoea, and infection with Trichomonas vaginalis, herpes simplex virus, and warts was almost unchanged. Other conditions, which are not classified as STD, were largely responsible for the increase in new attendances, the prevalence of these conditions having nearly doubled over the ten year period. Thus an epidemic of STD has not been shown in this adolescent female population over the past decade.

\section{Introduction}

Sexually transmitted diseases (STDs), especially gonorrhoea, have reached epidemic proportions in the United States of America, the incidence in 15-19 year old girls having increased fivefold during the decade 1965-75. ${ }^{1}$ In England and Wales, new cases of gonorrhoea in girls aged 16-19 increased by $22 \%$ during the decade $1970-80 . .^{23}$ The extent of the problem in both countries is not fully known as underreporting of cases occurs. Pelvic inflammatory disease (PID) is a serious consequence of STD. In England and Wales reported cases of PID in all age groups rose by $50 \%$ during the years $1968-77^{4}$; this is almost certainly an underestimate of cases. Furthermore, figures are based on hospital inpatient statistics alone. ${ }^{5}$ Data for 1982 are not yet available for comparison.

Details concerning the incidence of STD in adolescents in the United Kingdom are sparse, although national figures are available for syphilis and gonorrhoea in this age group. ${ }^{3}$ Hunter and Neilson were unable to show an epidemic of STD in their survey of all patients aged under 18 who attended an Edinburgh clinic in the years 1967 and $1977 .{ }^{6}$ The study reported here was therefore designed to

Address for reprints: Dr G E Forster, Senior Registrar, Praed Street Clinic, St Mary's Hospital, Praed Street, London W2 1NY

Accepted for publication 23 March 1984 compare the prevalence of STD in all adolescent girls, aged 15-19 attending the Praed Street Clinic, a large department of genitourinary medicine in west London, in the years 1972 and 1982. These years were selected because a substantial modification of the reporting of STD to the Department of Health occurred in 1971 with the addition of seven new categories, including herpes simplex and condyloma acuminatum, ${ }^{7}$ and this coding was established in the following year; furthermore, the most recent data available from the clinic were for 1982 .

\section{Patients and methods}

We studied the prevalence of STD in all adolescent girls aged 15-19 who attended this clinic in 1972 and 1982. Data were obtained from the confidential register using the coding of the clinic returns to the Department of Health (form SBH 60). Patients were counted whether or not they had attended in an earlier year. Each new episode in patients who attended more than once during the year was reported as a separate diagnosis. The numbers reported are thus closer to the concept of period prevalence than to incidence. Further information was obtained from the individual case records when necessary. Most patients were self referred; there was no appointment system, and all patients were seen on the day of presentation. Diagnoses were made according to recognised microbiological or clinical criteria, or both. ${ }^{8}$ 


\section{Results}

The study comprised 1373 patients in 1972 and 1799 in $1982,6.4 \%$ and $7 \%$ respectively of the total female clinic population in those years. Table I shows the number of patients in each age group for both years. Patients aged 18 and 19 comprised $75 \%$ (1972) and $80 \%$ (1982) of the groups studied.

TABLE I Age distribution of women attending an STD clinic in 1972 and 1982

\begin{tabular}{lrr}
\hline & \multicolumn{2}{c}{ No of patients attending in: } \\
\cline { 2 - 3 } Age (years) & 1972 & 1982 \\
\hline 15 & 20 & 12 \\
16 & 123 & 94 \\
17 & 202 & 257 \\
18 & 449 & 554 \\
19 & 579 & 882 \\
Total & 1373 & 1799 \\
\hline
\end{tabular}

Self referral increased from $65 \%$ of patients in 1972 to $74 \%$ of those in 1982 . The number of cases exceeded the number of patients as an average of 1.2 diagnoses per patient was made in each year. More than one specific diagnosis of STD was made in $14 \%$ (1972) and $8.4 \%(1982)$ of the patients. Table II shows that the prevalence of syphilis, gonorrhoea, trichomonal vaginitis, infection with herpes simplex virus (HSV), and genital warts was almost the same in the two years.

TABLE II Diagnoses recorded for women attending an STD clinic in 1972 and 1982

\begin{tabular}{lrr}
\hline & \multicolumn{2}{c}{ No of diagnoses in: } \\
\cline { 2 - 3 } Diagnosis & 1972 & 1982 \\
\hline Syphilis & 2 & 6 \\
Gonorrhoea & 247 & 287 \\
Trichomoniasis & 294 & 244 \\
Candidiasis & 336 & 238 \\
Scabies & 14 & 5 \\
Pubic lice & 28 & 25 \\
Herpes simplex virus & 44 & 60 \\
infection & 124 & 141 \\
Warts & 5 & 2 \\
Molluscum contagiosum & 1094 & 1008 \\
Total & &
\end{tabular}

\section{SYPHILIS}

Two cases of syphilis were diagnosed in 1972 and six in 1982 in the groups studied. Two patients presented with secondary syphilis in 1982 . The six latent cases were diagnosed by serological tests.

\section{GONORRHOEA}

The prevalence of gonorrhoea declined from $18 \%$ (1972) to $16 \%$ (1982). There were seven cases of infection with $\beta$-lactamase producing Neisseria gonorrhoeae in 1982.

\section{NON-SPECIFIC GENITAL INFECTION}

Non-specific genital infection (NSGI) is not recognised as a separate clinical entity in women attending the Praed Street Clinic. No diagnostic service for the isolation of Chlamydia trachomatis was available during the decade. Contacts of men with non-gonococcal urethritis (NGU) were classified as having "other conditions not requiring treatment" or, when treated epidemiologically, as having "other conditions requiring treatment". In 1972, 123 patients in the study population were contacts of men with NGU. Of these, 77 were treated epidemiologically and were classified as having "other conditions requiring treatment". The remaining 46 contacts were coded as having "other conditions not requiring treatment" and were not treated.

\section{HERPES SIMPLEX VIRUS INFECTION}

The number of cases of infection with HSV increased from 44 (1972) to 60 (1982), which represented 20\% and $17 \%$ respectively of all women infected with HSV in those years. Diagnosis was based on clinical impression in 1972, but was supported by viral culture in most cases (42 of 60) in 1982.

\section{GENITAL WARTS}

The prevalence of genital warts declined from $9 \%$ (1972) to $8 \%$ (1982). Diagnosis of this condition remained unchanged over the decade.

\section{OTHER STDS}

The prevalence of the remaining STDs including candidiasis, scabies, pubic lice, molluscum contagiosum, and trichomonal vaginitis was lower in 1982 than in 1972. Diagnostic techniques for these conditions were unchanged.

\section{OTHER CONDITIONS}

Table III shows that the largest absolute increase in attendances over the decade was in the diagnostic category "other conditions" excluding syphilis,

TABLE III Number of patients with "other conditions" in 1972 and 1982

\begin{tabular}{llr}
\hline & \multicolumn{2}{l}{ No of diagnoses in: } \\
\cline { 2 - 3 } Diagnosis & 1972 & 1982 \\
\hline $\begin{array}{l}\text { Other conditions } \\
\text { requiring treatment }\end{array}$ & 387 & 658 \\
$\begin{array}{l}\text { Other conditions not } \\
\text { requiring treatment }\end{array}$ & 226 & 430 \\
$\begin{array}{l}\text { Other conditions } \\
\text { referred elsewhere }\end{array}$ & 613 & 12 \\
Total & & 1100 \\
\hline
\end{tabular}


gonorrhoea, and other recognised STDs, which represented $45 \%$ (1972) and $61 \%$ (1982) of the study populations. Table IV is an analysis, using problem orientated categories, of the miscellaneous collection of diagnoses which comprise "other conditions".

TABLE IV Analysis of "other conditions" diagnosed

\begin{tabular}{lcr}
\hline & \multicolumn{2}{c}{ No of diagnoses in: } \\
\cline { 2 - 3 } Diagnosis & 1972 & 1982 \\
\hline Epidemiological treatment of: & 2 & \\
$\quad$ Contacts of syphilis & 69 & 70 \\
Contacts of gonorrhoea & 77 & 117 \\
$\quad$ Contacts of non-gonococcal & 148 & 187 \\
$\quad$ urethritis & 189 & 448 \\
Total & 5 & 22 \\
Lower genital tract symptoms & 19 & 39 \\
Upper genital tract symptoms & 12. & 57 \\
Urinary tract symptoms & 78 & 186 \\
Gynaecological symptoms & $162^{*}$ & 161 \\
Asymptomatic (patients & 613 & 1100 \\
requesting check up) & Miscellaneous & \\
Total &
\end{tabular}

*This figure includes 46 patients who were contacts of men with NGU considered to have "other conditions not requiring treatment".

\section{Discussion}

The 1981 STD surveillance report commented on the increasing importance of new cases of STD in categories other than syphilis or gonorrhoea in all age groups in the United Kingdom. ${ }^{9}$ The incidence of gonorrhoea reached a peak in the mid-1970s and has declined since 1977.5 The number of reported cases of non-specific genital infection has, however, continued to rise. ${ }^{9}$ Belsey concluded that national statistics for cases of NSGI in women were virtually worthless because of variations in criteria of diagnosis and notification. ${ }^{10}$ Despite the current media interest in HSV infection, this was responsible for only $\mathbf{2} \cdot \mathbf{3 \%}$ of all new cases seen in STD clinics in $1981 .{ }^{9}$ There were fewer cases of scabies and infection with $T$ vaginalis in the latest report compared with that for preceding years. ${ }^{9}$ The largest absolute increase in new attendances in 1981 by diagnostic category was in "other conditions requiring treatment". Thus national data suggest that cases of NSGI and "other conditions requiring treatment", rather than syphilis, gonorrhoea, and other recognised STDs, are responsible for the increasing attendance at clinics in the United Kingdom, which contrasts with American data.

The national data are supported by this study, which shows that there has not been an epidemic of STD in adolescent girls attending this clinic. The prevalence of all STDs in the study population declined from $80 \%$ in 1972 to $56 \%$ in 1982 . Syphilis and gonorrhoea together were responsible for $18 \%$ (1972) and 16\% (1982) of all new cases requiring treatment. NSGI is not recognised as a separate entity in women attending this clinic because, as suggested by Belsey, ${ }^{10}$ acceptable and reproducible criteria for the diagnosis of NSGI are not available. The total number of patients attending as known contacts of men with NGU declined from 123 in 1972 to 117 in 1982 . Most were treated epidemiologically and classified as having "other conditions requiring treatment". In 1972, when epidemiological treatment was not yet established clinic practice, 46 of them were not treated and were diagnosed as having "other conditions not requiring treatment". It is possible that some cases of chlamydial infection remained undiagnosed in both 1972 and 1982 in the absence of a culture service for this microorganism. ${ }^{11}$

Patients and doctors had a greater awareness of infection with HSV by 1982 . The prevalence of such infection, however, remained constant at $3 \%$ of all new cases in the study populations in both years. A clinical diagnosis of HSV infection was acceptable in 1972 when viral culture services were limited. By 1982 , isolation of the virus was the method of choice in the diagnosis of infection with HSV, and gave positive results in 42 of the 60 cases. A clinical diagnosis was made when a patient attended too late in the course of the infection to give a positive culture. A review of patients classified as having "other conditions requiring or not requiring treatment" did not show any additional cases of genital ulceration that might have been undiagnosed infection with HSV in either year.

Diagnostic criteria for the other recognised STDs have remained unchanged over the decade. Nevertheless, in all categories there was a reduction in prevalence of STD between 1972 and 1982 .

Despite the decline in prevalence of STD in the girls studied, clinic attendances have increased by $31 \%$ over the decade. Attendances for conditions not classified as STD were responsible for this increase, these having nearly doubled over the years 1972-82 (table III). Further clarification of the diagnostic group "other conditions" is required. ${ }^{1012}$ This consists of a miscellaneous collection of diagnoses including those requiring epidemiological treatment, for which no standard notification exists (table IV). Epidemiological treatment for contacts of men with syphilis, gonorrhoea, and NGU is established clinical practice and accounted for $10 \%$ of the attendances. Furthermore, infection with other micro-organisms, such as Gardnerella vaginalis whose pathogenicity is yet to be ascertained, ${ }^{13}$ is currently recorded in this category. In 1982 this condition accounted for 191 $(10 \cdot 6 \%)$ of the attendances of women studied. 
The increased numbers of attendances of girls in 1982 was therefore accounted for by attendances of those in whom no STD could be found. A decline in the prevalence of STD was also noted.

We thank Drs J R W Harris and D Goldmeier for allowing us to review patients under their care, and Dr D Hall, Director, Community Medicine and Nursing Research Unit, St Mary's Hospital, London W2 1NY for help in analysing the data. We also thank Mrs K English and the reception staff of the Praed Street Clinic for their help.

\section{References}

1. Shafer M-AB, Irwin CE, Sweet RL. Acute salpingitis in the adolescent female. J Pediatr 1982; 100:339-50.

2. Department of Health and Social Security. Sexually transmitted diseases. In: On the state of the public health. The annual report of the Chief Medical Officer of the Department of Health and Social Security for the year 1971. London: HMSO, 1972:69.
3. Department of Health and Social Security. Sexually transmitted diseases. In: On the state of the public health. The annual report of the Chief Medical Officer of the Department of Health and Social Security for the year 1981. London: HMSO, 1982:53.

4. Catterall RD. Biological effects of sexual freedom. Lancet 1981;i:315-9.

5. Anonymous. Sexually transmitted disease surveillance 1979. $\mathrm{Br}$ Med J 1981;282: 155-6.

6. Hunter JM, Neilson M. Sexually transmitted diseases in Edinburgh: patients under 18 years of age. Health Bull (Edinb) $1980 ; 38: 23-8$.

7. Anonymous. Sexually transmitted disease surveillance 1978. $\mathrm{Br}$ Med J 1979; ii: 1375-6.

8. Schofield CBS. Sexually transmitted diseases. 3rd ed. Edinburgh: Churchill Livingstone, 1979.

9. Anonymous. Sexually transmitted disease surveillance 1981. Br Med J 1983; 286: 1500-1.

10. Belsey EM. Epidemiological treatment of gonorrhoea and nonspecific genital infection in female sexual contacts. Br J Vener Dis 1982;58: 113-6.

11. Munday PE, Pritchard G, Harris JRW, Taylor-Robinson D. Prevalence of chlamydial infection in promiscuous women. $B r$ J Vener Dis 1983;59:103-4.

12. Adler MW. Current routine statistics in the United Kingdom: room for improvement? Br J Vener Dis 1981;57:83-8.

13. Dawson SG, Harris JRW. Gardnerella vaginalis and nonspecific vaginitis. Br J Hosp Med 1983; 29:28-37. 